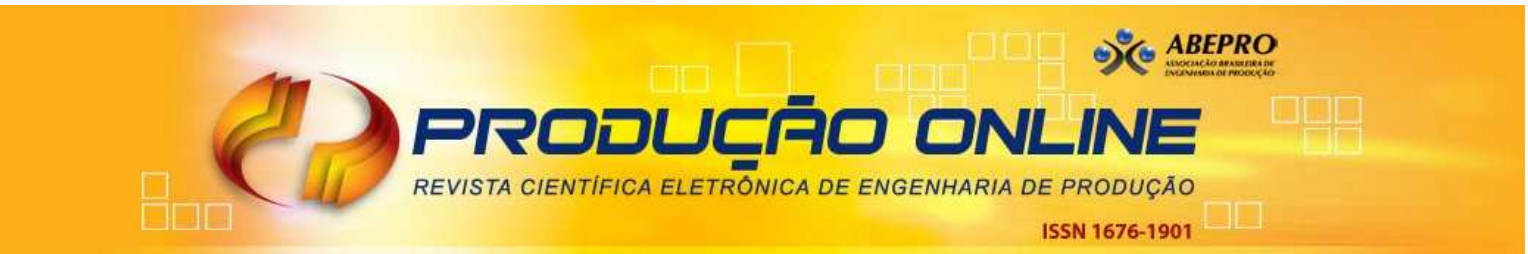

\title{
AVALIAÇÃO DE PROJETO PARA IMPLANTAÇÃO DE UM SISTEMA DE VOIP: UM CASO NA INDÚSTRIA DE ALIMENTOS
}

\section{ASSESSMENT OF AN IMPLEMENTATION PROJECT FOR A VOIP SYSTEM: A CASE IN THE FOOD INDUSTRY}

\author{
Tiago José Menezes Gonçalves* E-mail: tiagojmg@ita.br \\ Armando Gonçalves Madeira-Junior* E-mail: madeira@ita.br \\ Alcimar das Chagas Ribeiro** E-mail: alcimar@uenf.br \\ * Instituto Tecnológico de Aeronáutica - ITA, São José dos Campos, SP \\ ** Universidade Estadual do Norte Fluminense - UENF, Campos dos Goytacazes - RJ
}

\begin{abstract}
Resumo: No século XXI, todas as organizações são afetadas direta ou indiretamente pelo uso das tecnologias da informação e comunicação (TIC). A elaboração de bons projetos para implantação dessas tecnologias é de fundamental importância para que uma empresa se mantenha competitiva frente a seus concorrentes. Neste contexto, este trabalho analisa a viabilidade econômica para a implantação de um sistema de comunicação baseado em VolP (Voice on Internet Protocol) em uma empresa do setor industrial alimentício. Neste sentido, foram comparados o sistema de telefonia existente na empresa com um projeto proposto para implantação de um sistema de VolP. Para tanto, foi realizada a modelagem dos custos incorridos através de equações e utilizados como indicadores de viabilidade do projeto a análise do ponto de equilíbrio, o payback, o valor presente líquido e a taxa interna de retorno. Como resultado, concluiu-se que a implantação de sistemas de telefonia baseados em VolP pode resultar em grande economia de custos, permitindo recuperar rapidamente o capital investido no projeto. Esta conclusão foi ratificada na análise de todos os indicadores citados acima, o que permitiu recomendar a implementação do projeto e verificar a aplicabilidade dos modelos de custo desenvolvidos.
\end{abstract}

Palavras-Chave: Viabilidade Econômica de Projetos. Integração da Cadeia de Suprimentos. Tecnologia da Informação e Comunicação. VoIP. Indústria de Alimentos.

\begin{abstract}
In the twenty-first century, all organizations are affected directly or indirectly by the use of information technology and communication (ICT). The preparation of good projects to implement these technologies is relevant for a company to remain competitive compared to its competitors. In this context, this study analyzes the economic viability for the implementation of a communication system based on VolP (Voice on Internet Protocol) in a food company of the industrial sector. In this way, we compared the existing telephony system in the company with a proposed project for implementation of a VolP system. Therefore, we performed the modeling of costs incurred through of equations and we used as indicators of project viability the break-even point analysis, the payback period analysis, the net present value and the internal rate of return. As result, it was concluded that the implementation of VolP-based systems can result in large cost saving, allowing rapidly recovering of the capital invested in the project. This conclusion was confirmed by the analysis of all the indicators cited above, which allowed to recommend the implementation of the project and verify the applicability of cost models developed.
\end{abstract}

Keywords: Economic Viability of Projects. Integration of Supply Chain. Information Technology and Communication. VolP. Food Industry 


\section{INTRODUÇÃO}

As grandes transformações introduzidas pelas tecnologias da informação e comunicação (TIC) mudaram radicalmente a maneira como as organizações realizam seus negócios. As inovações introduzidas por essas tecnologias possibilitaram grande melhora de desempenho, e se firmaram como requisitos básicos para a sobrevivência da maioria das empresas.

Segundo Laudon (2004), as empresas integrantes de um setor podem utilizar as tecnologias da informação e comunicação para desenvolver padrões de troca de informações, o que força os participantes do mercado a aderirem a padrões semelhantes para continuarem competitivos.

$O$ entrosamento entre os elos da cadeia de suprimentos desempenha um papel preponderante para o sucesso de uma empresa (SANTOS, 2010). No caso particular da indústria de alimentos, devido à sazonalidade e a perecibilidade dos insumos que utiliza este fator torna-se ainda mais crítico. Para atingir tal entrosamento, modernos sistemas de informações podem ser utilizados para possibilitar a troca de informações de forma mais eficiente ao longo de toda cadeia (TURBAN, 2005).

De acordo com a Associação Brasileira das Indústrias de Alimentação - ABIA (2008), a indústria de alimentos tem expressiva participação na economia brasileira, contribuindo com cerca de 9,3\% do Produto Interno Bruto (PIB), e correspondendo a aproximadamente $18 \%$ da participação da indústria de transformação do país. Segundo a mesma associação, o setor é composto de um grande número de micro e pequenas indústrias (que correspondem a $94,7 \%$ do total) que atuam geralmente em mercados regionais, e que têm grande importância local na absorção de mão de obra e na geração de riquezas. Como o próprio nome diz, as micro e pequenas empresas do setor industrial alimentício apresentam baixa escala de produção, com produtos geralmente de baixa sofisticação tecnológica (muitas vezes artesanais) e freqüentemente ligados à cultura local. Trata-se de um setor produtivo com uma extensa cadeia de suprimentos, que envolve elos de várias cadeias produtivas desde a agricultura e pecuária até o consumidor final.

Embora a literatura de sistemas de informação apresente diversos trabalhos preocupados com projetos de implantação de TIC, poucos são aqueles que 
focalizam o setor industrial alimentício do ponto de vista da integração de sua cadeia de suprimentos, sendo que pesquisas nesta área são muito esparsas ou mesmo inexistentes, principalmente quando se trata de micro e pequenas empresas (MPEs).

O objetivo deste trabalho é analisar a importância da análise de viabilidade econômica de projetos para implantação de TIC em MPEs do setor mencionado, ao mesmo tempo em que apresenta como o uso das TIC pode fornecer vantagem competitiva a uma empresa do setor ao incrementar a integração da cadeia de fornecimento e/ou reduzir seus custos de comunicação. Para alcançar tal objetivo, foi conduzido um estudo de caso de forma a auxiliar na compreensão sobre os fatores mais relevantes na análise da viabilidade econômica de um projeto, discutindo como esta análise pode contribuir para o sucesso da implantação de TIC nessas empresas e no entendimento de como tais empresas têm feito a avaliação de seus investimentos nesta área.

Este artigo está estruturado no seguinte formato: na seção1, o trabalho é apresentado, bem como seu contexto e relevância; a seção 2 trata da fundamentação teórica relacionada à importância dos projetos de TIC, da análise de viabilidade desses projetos, da Internet como meio de comunicação e de como a TIC pode influenciar na integração da cadeia de suprimentos da indústria de alimentos; a seção 3 trata da metodologia utilizada no estudo; a seção 4 apresenta a unidade de análise do estudo de caso, seguida da descrição do problema empresarial abordado no estudo e do projeto de TIC proposto para solucioná-lo; na seção 5 é realizada a análise de viabilidade do projeto, com a descrição do projeto e a utilização de métodos quantitativos de análise financeira para o estudo de viabilidade econômica; por último, a seção 6 apresenta as considerações finais do presente trabalho.

\section{FUNDAMENTAÇÃO TEÓRICA}

\subsection{Projetos de implantação de TIC}

A implantação de TIC pode envolver a utilização de grandes volumes de recursos, constituindo profundas mudanças numa organização. Para que a direção de uma empresa se comprometa com a direção de um projeto, são necessárias 
justificativas sólidas, que podem ser obtidas através de uma análise de viabilidade econômica.

Em 1994, Strassmann (1997) analisou 539 empresas dos Estados Unidos, Canadá e Europa, e verificou que não existe qualquer correlação entre a intensidade de investimentos em TIC e o retorno obtido pela empresa. Com este resultado, o autor mencionado derrubou o mito de que as empresas que investem mais em TIC obtêm necessariamente o melhor resultado.

Este fato ressalta a necessidade de formas adequadas para se avaliar os investimentos em TIC, que sejam capazes de direcionar o processo de tomada de decisão sobre o investimento e que não fiquem restritas apenas a intuição dos tomadores de decisão.

Segundo Colangelo Filho (2001), uma análise de viabilidade econômica é o trabalho que avalia uma proposta de projeto, concluindo sobre sua aceitação ou rejeição. De acordo com o mesmo autor, existem três motivos para elaborar um estudo de viabilidade econômica em um projeto para implantação de TIC, a saber:

- Para dar suporte a uma eventual implantação do sistema;

- Para identificar os benefícios e metas, mantendo os responsáveis cientes sobre os objetivos do projeto, para que os mesmos possam ser alcançados;

- Para identificar os recursos necessários para a implantação do projeto, permitindo solicitar o comprometimento da organização com a mobilização deles.

Constata-se que o estudo da viabilidade econômica de um projeto é de fundamental importância para uma organização, e deve ser utilizado para auxiliar na decisão de investir de modo a minimizar os riscos da decisão. Alguns dos métodos mais utilizados estão apresentados na próxima seção.

\subsection{Métodos de análise de viabilidade econômica}

De acordo com Britto (2004) entre os principais métodos de análise de viabilidade econômica estão o Valor Presente Líquido (VPL), o Payback, a Análise do Ponto de Equilíbrio e a Taxa Interna de Retorno (TIR).

Segundo Peres et al. (2004) o VPL corresponde à soma algébrica dos valores do 
fluxo de caixa do projeto em análise, atualizados à taxa de desconto do período avaliado, sendo um projeto considerado viável quando apresentar um VPL positivo.

Neste sentido, na seleção de projetos, escolhe-se aquele que apresentar o maior valor positivo para esse indicador. Seu cálculo é realizado conforme a Equação 1.

$$
\mathrm{VPL}=\sum_{t=0}^{n} \operatorname{VFL} /(1-r)^{t}
$$

Onde:

$\mathrm{VPL}=$ Valor Presente Líquido;

$\mathrm{VFL}=$ Valor do Fluxo Líquido (diferença entre entradas e saídas de caixa);

$r=$ Taxa de desconto;

$\mathrm{n}=$ Número de fluxos;

$\mathrm{t}=$ Número de períodos.

Em relação ao Payback (também conhecido como Tempo de Amortização do Investimento), conforme afirmam Bruni, Famá e Siqueira (1998) este método consiste em analisar o tempo necessário para que todo o capital investido no projeto seja integralmente recuperado. Quanto menor for o payback encontrado nesta análise, menor é o risco do projeto e mais rapidamente o capital é retornado para ser reinvestido. $O$ cálculo do payback é realizado conforme Equação 2.

$$
\text { Payback }=\frac{\text { Investimento }}{\text { Retorno/ período }}
$$

Onde:

Investimento $=$ Total investido no projeto;

Retorno/período = Retorno líquido de caixa por período.

A Análise do Ponto de Equilíbrio consiste em analisar a escala de utilização em que os custos de um ou mais projetos se igualam, onde são considerados lucrativos os investimentos que se encontram acima do ponto de equilíbrio (BRITTO, 2004).

Por fim, a Taxa Interna de Retorno (TIR) consiste na taxa de desconto que torna o Valor Presente Líquido do investimento (VPL) nulo. Para este indicador, devem ser escolhidos os projetos que apresentem as maiores TIR, sendo quanto maior este indicador, mais desejável o investimento, sendo seu cálculo realizado conforme a Equação 3 (WOILER; MATHIAS, 1996). 


$$
\sum_{t=0}^{n} V F L /(1-r)^{t}=0
$$

\subsection{Cadeia de suprimentos e a indústria de alimentos}

De acordo com Gaither e Frazier (2002), uma cadeia de suprimentos, em seu escopo mais amplo, refere-se à maneira pela qual os materiais fluem através de diferentes organizações, iniciando com as matérias-primas e encerrando com produtos acabados entregues ao consumidor final. Ela é uma junção de fornecedores, distribuidores, fabricantes, clientes e os clientes dos clientes.

O fluxo de informações é de grande importância para todas as operações de uma cadeia de suprimentos. A transferência eletrônica de informações proporciona uma oportunidade de reduzir custos, aperfeiçoar o serviço e melhorar a oferta de informações, mediante a melhor coordenação da rede de operações.

Para Dias et al. (1997), uma estratégia competitiva desenvolvida para capturar e maximizar as oportunidades das cadeias de suprimento exige que todas as empresas participantes trabalhem em perfeita sincronia, numa estrutura de redes dinâmicas. Para alcançar tal sincronia entre as operações de uma rede produtiva, muitas empresas estão fazendo uso das TIC para alcançar benefícios tais como a melhoria dos canais de comunicação com clientes e fornecedores e a agilização nas operações de compra e venda, aumentando a competitividade empresarial (LA ROVERE, 1999).

Como pode ser verificada na Figura 1, a cadeia de suprimentos da indústria de alimentos abrange desde o fornecimento de insumos genéricos da agroindústria até a distribuição para o consumidor final. Tendo em vista que os insumos utilizados em sua produção são perecíveis, constata-se que o sucesso da indústria de alimentos está fortemente ligado ao grau de integração entre os elos da cadeia de fornecimento da qual participa, pois seu processo de transformação depende diretamente da qualidade dos insumos utilizados.

O grau de integração de uma cadeia de suprimentos é definido como o nível de conexão entre cada operação de uma rede (LI, 2002; WOOD, 1997; NARASIMHAN; JAYARAM, 1998). Segundo Lambert e Cooper (2000), o grau de integração de uma cadeia de suprimentos é uma função do número de componentes de gestão existente em cada elo da rede, sendo que quanto maior esse número de 
componentes, maior é considerado o grau de integração em uma cadeia de suprimentos. Em meio a esses diversos componentes de gestão, pode-se destacar a estrutura para fluxo de produtos e a estrutura para fluxos de informações, que são componentes críticos para o sucesso das indústrias de alimentos.

Analisando-se os benefícios obtidos com a utilização de ferramentas de TIC, tais como o maior intercâmbio de informações, a redução de custos e a maior velocidade de comunicação; verifica-se que essas ferramentas são de grande valor para a integração da cadeia de suprimentos de qualquer empresa, possibilitando a dinamização do fluxo de informações e aumentando a sinergia entre as operações de uma rede, contribuindo intensamente para o sucesso de uma operação.

Figura 1 - Cadeia produtiva do setor industrial alimentício

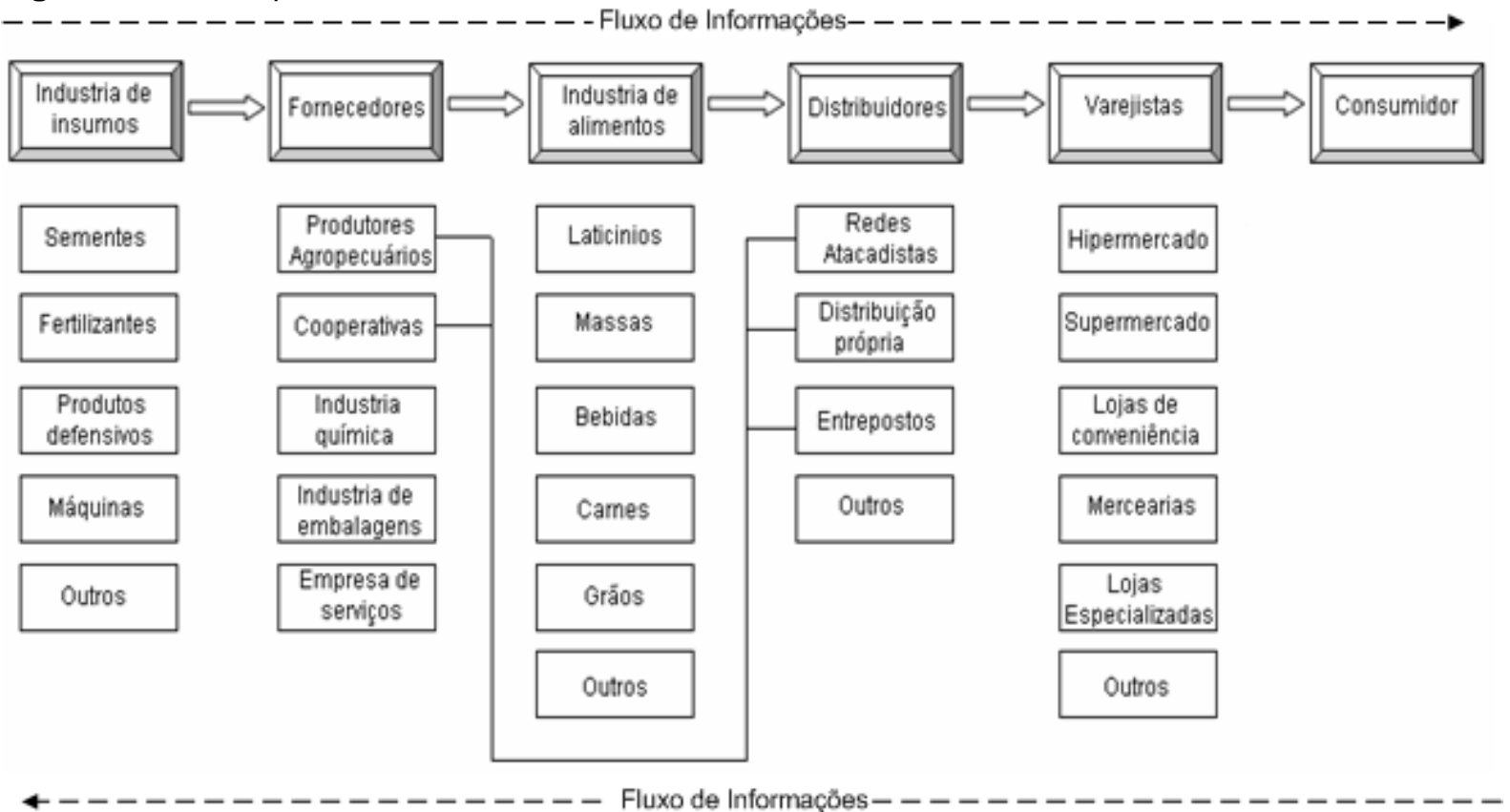

Fonte: Elaborada pelos autores

\subsection{Internet e Comunicação}

De acordo com Ruthfield (2001) a Internet surgiu na década de 1970, durante a Guerra Fria, como resposta a preocupações do governo americano sobre a forma como deveriam ser as comunicações caso houvesse uma guerra nuclear. Nessa situação, os sistemas centralizados tradicionais não funcionariam, pois poderiam ser facilmente destruídos. Durante essa década a Internet possibilitou a comunicação entre diversos centros de pesquisa dos Estados Unidos. 
$\mathrm{Na}$ década de 1980 a Internet foi expandida para outros países, e passou a utilizar a tecnologia denominada Transmission Control Protocol/Internet Protocol (TCP/IP), que é mais adequada para redes com grande número de servidores. No início da década de 1990 foi criada a World Wide Web, também denominada de WWW ou Web (BERNERS-LEE et al., 1994). Neste período a Internet distribuiu-se pelo mundo todo com a disseminação da microinformática, tendo sua utilização estendida para fora do âmbito universitário (CASTELLANI, 1998).

É importante entender os diferentes significados entre Web e Internet. Segundo Oggh (2001), "a Web é um serviço que utiliza a estrutura da Internet", enquanto a Internet "é apenas um meio que permite que computadores conversem entre si através de um protocolo chamado TCP/IP". Ou seja, o usuário não acessa a Internet, e sim um serviço através dela.

No Brasil, a implantação da Internet teve início em 1987, e somente a partir de 1995 ela foi disponibilizada para exploração comercial (FONTES, 2001). De 1995 até hoje a Internet continuou crescendo, sendo que até o fim de 2008 existiam no Brasil mais de 60 milhões de usuários da rede (DIAS, 2009).

Atualmente a Web é utilizada pelas organizações como uma ferramenta para a realização de processos de forma mais eficiente, sendo extensivamente utilizada como o principal meio de comunicação com o mundo externo. Seu desenvolvimento teve um impacto significativo na maneira como as organizações realizam seus negócios, permitindo a criação de novos produtos de comunicação e a redução de custos (GONÇALVES, 2010).

Neste ambiente de desenvolvimento das tecnologias de comunicação, através da Internet que viabilizou a criação de uma tecnologia para transmissão de áudio digital em tempo real, sendo esta tecnologia denominada de VoIP (Voice on Internet Protocol) (MONTEIRO, 2000).

Por fim, cabe ressaltar que soluções baseadas em Internet Protocol (IP) têm sido propostas para substituir os modelos de telefonia convencional com inúmeras vantagens (CUNHA, 2004). Neste sentido, este trabalho investiga as vantagens da comunicação por VolP no que se refere à custos, conforme é abordado nas próximas seções. 


\section{METODOLOGIA}

O presente trabalho enquadra-se na categoria denominada estudo de caso. Jung (2004) define estudo de caso como sendo um procedimento de pesquisa que investiga um fenômeno dentro de um contexto local e real, sobretudo quando as fronteiras entre o fenômeno e o contexto não estão claramente definidas.

Para Godoy (1995), a principal característica de um estudo de caso é estudar de forma intensiva e detalhada uma entidade bem definida, que pode ser uma organização, um processo, uma política, um método de trabalho, um contexto, um grupo de indivíduos, uma comunidade, etc.

No campo da gestão empresarial, uma das finalidades do estudo de caso é apresentar práticas adequadas de gestão que estão acontecendo nas organizações. A descrição de uma prática adequada em um estudo de caso permite o fornecimento de informações para situações similares, compartilhando as conquistas obtidas com outras pessoas ou empresas, que podem aprender e reaplicar estas informações em seu contexto.

Neste trabalho foi realizado um estudo de forma a analisar a importância da análise de viabilidade econômica de projetos de TIC, ao mesmo tempo em que apresenta como o uso da TIC pode ajudar na integração da cadeia de fornecimento de uma empresa do setor industrial alimentício. Para alcançar tal objetivo foi conduzido um estudo destinado a analisar a viabilidade econômica de um projeto de TIC para a substituição de um sistema de comunicação através de linha telefônica (existente na empresa analisada) por um sistema de comunicação por VoIP.

Para a realização da análise de viabilidade econômica, foram coletados os dados referentes aos custos de utilização de ambos os sistemas analisados, de implantação do sistema proposto (VoIP) e do perfil de utilização deste tipo de sistema pela empresa. Os métodos de coleta de dados utilizados foram dois:

- Entrevista: Neste trabalho foram entrevistados os três gerentes da empresa estudada, os quais informaram o perfil de utilização das tecnologias de comunicação por voz na empresa. Através deste método foram obtidos dados a respeito do tipo de plano de tarifação telefônica utilizada pela empresa, o percentual aproximado de cada tipo de ligação 
realizada (ligação local, DDD, DDI, etc.) e para quais tipos de aparelhos essas ligações eram destinadas (telefones fixos ou celulares);

- Pesquisa na Internet: Para a realização da análise de viabilidade econômica e a comparação entre os dois sistemas de comunicação analisados neste trabalho, foi realizada uma pesquisa na Internet sobre a tarifação destes serviços e sobre o valor dos equipamentos cuja compra seria necessária no caso da implantação do sistema de comunicação via VolP. Esta pesquisa consistiu em acessar os web sites das operadoras dos sistemas analisados e de lojas on-line, e consultar as tarifas e os valores dos equipamentos necessários para a realização da análise de viabilidade econômica.

Após a coleta de dados sobre os custos de utilização e/ou implantação de ambos os sistemas, foi realizada a análise de viabilidade econômica para a implantação do novo sistema de telefonia via VoIP, conforme os resultados apresentados mais adiante neste trabalho.

\section{UNIDADE DE ANÁLISE}

A empresa analisada atua na produção de doces de goiaba, banana e melados, estando a trinta e seis anos no mercado. A empresa emprega 29 funcionários e tem um faturamento anual de aproximadamente 120 mil reais. Ela é formada por uma unidade industrial localizada na Região Norte Fluminense - RJ, e comercializa seus produtos em toda Região Sudeste.

\subsection{Identificação e diagnóstico do problema}

O problema abordado neste trabalho foi à ineficiência de comunicação entre a empresa e os demais elos de sua cadeia produtiva devido à precariedade dos meios de comunicação utilizados.

O telefone e a Internet são os principais meios de comunicação da empresa, sendo que a linha telefônica é utilizada para o acesso a internet. Devido à velocidade baixa do acesso discado, a navegação pela internet é lenta e o uso simultâneo da internet e do telefone é impossibilitado. 
O problema de comunicação é especialmente grave por se tratar de uma indústria de alimentos, onde os insumos para a produção fabril são perecíveis e os meios para sua conservação são onerosos, o que obriga a busca de uma maior integração da empresa com sua cadeia de fornecimento.

\subsection{Projeto para implantação de TIC}

A solução encontrada para o problema de comunicação descrito na seção anterior foi o cancelamento da linha telefônica e a utilização de um programa de VolP com a implantação de internet de banda larga.

O primeiro benefício obtido com este projeto trata-se de passar a usufruir de internet de banda larga, o que proporciona maior velocidade de acesso aos recursos da internet utilizados pela empresa, como a utilização de correio eletrônico, messenger instantâneo e outros serviços que a empresa poderia vir a utilizar.

O segundo benefício trata-se do menor custo de comunicação por voz. Comunicando-se com telefones externos utilizando-se um programa de VoIP, a empresa arcaria com tarifas significativamente menores do que com o uso da linha telefônica. Além do mais, para fazer uma ligação entre dois computadores utilizando o mesmo programa de VoIP a tarifa cobrada é zero, favorecendo a informatização conjunta dos elos da cadeia de suprimentos para desfrutar de gratuidade na comunicação com as outras operações da cadeia produtiva. Uma análise completa sobre a viabilidade econômica essa deste projeto é realizada na seção seguinte.

Uma das dificuldades encontradas nesta proposta foi o fato de que o estabelecimento estudado situa-se numa região afastada da cidade, onde aparentemente não há nenhum canal de conexão com internet de banda larga (a cabo, a rádio, etc.). Este obstáculo foi superado com a percepção de que existe sinal de telefonia móvel $3 G$ da empresa Claro de telefonia móvel, que permite acesso à internet com opções de velocidade entre $250 \mathrm{kbps}$ e $1 \mathrm{mbps}$, bastando a conexão com um modem USB. 


\section{ANÁLISE DE VIABILIDADE ECONÔMICA DO PROJETO}

\subsection{Modelos de custos para simulação}

Nesta análise, foi adotado o plano Claro 3G (EMPRESA CLARO DE TELEFONIA, 2009) com velocidade de 1 mbps e acesso ilimitado a internet, com tarifa de $\mathrm{R} \$ 99,99$. Os planos com tarifas menores foram desconsiderados por não atenderem os requisitos da empresa em termos de velocidade. O programa de VoIP adotado neste trabalho foi o Skype. Para receber chamadas de telefones externos diretamente no Skype, é necessária a compra de um número de telefone local utilizando o serviço Skypeln, no valor de $\mathrm{R} \$ 80,00$ anuais. Além do custo com o Skypeln, toda vez que for realizada uma ligação, será cobrada a tarifa de conexão de $\mathrm{R} \$ 0,09$ (uma única vez).

Em relação à utilização da linha telefônica, a tarifa de assinatura mensal da linha é de $R \$ 41,03$. A empresa utiliza os serviços da Telemar para ligações locais e o serviço da Embratel para ligações à distância (este último com o plano Sim 21 Plus, que tem as mesmas tarifas durante todo o horário comercial). Na Tabela 1 estão listadas as tarifas cobradas pela utilização da linha telefônica e pelo sistema Claro 3G com Skype.

Os gerentes da empresa informaram os seguintes dados sobre as ligações realizadas:

- $70 \%$ das ligações são feitas para telefones fixos, das quais $80 \%$ são ligações locais;

- Das ligações feitas para telefones celulares, $90 \%$ são ligações locais;

- A empresa realiza em média 1300 ligações por mês, que correspondem a uma média de 3600 minutos por mês em ligações. 
Tabela 1 - Tarifas cobradas no Skype e no telefone fixo.

\begin{tabular}{lcc}
\hline \multicolumn{1}{c}{ Tipo de ligação } & \multicolumn{2}{c}{ Valor da tarifa/minuto } \\
\cline { 2 - 3 } & Skype & Linha telefônica \\
\hline Ligação local para telefone fixo & $\mathrm{R} \$ 0,08$ & $\mathrm{R} \$ 0,10$ \\
Ligação à distância para telefone fixo & $\mathrm{R} \$ 0,08$ & $\mathrm{R} \$ 0,45$ \\
Ligação local para telefone celular & $\mathrm{R} \$ 0,52$ & $\mathrm{R} \$ 0,81$ \\
Ligação à distância para telefone celular & $\mathrm{R} \$ 0,52$ & $\mathrm{R} \$ 1,73$ \\
\hline
\end{tabular}

Fonte: Adaptado de Embratel (2009) e Skype (2009).

Com base nas tarifas da Tabela 1 e na distribuição das ligações acima, foram desenvolvidos dois modelos para previsão dos custos mensais que a empresa teria com a comunicação por voz utilizando os dois sistemas. Os modelos descritos a seguir descrevem os custos variáveis em função do tempo de utilização (t).

No caso da linha telefônica, o custo de assinatura da linha dá direito a empresa a um tempo de 200 minutos de ligações locais para telefone fixo (LF). Dessa forma, foi desenvolvido um modelo para LF $<200$ minutos e para LF $\geq 200$ minutos.

Para LF $<200$ minutos:

$$
c(t)=41,03+(0,7 \times 0,2 \times 0,45) t+(0,3 \times 0,9 \times 0,81) t+(0,3 \times 0,1 \times 1,73) t
$$

Para LF $\geq 200$ minutos:

$c(t)=41,03+(0,7 \times 0,8 \times 0,10)(t-200)+(0,7 \times 0,2 \times 0,45) t+(0,3 \times 0,9 \times 0,81) t+(0,3 \times 0,1 \times 1,73) t$ Resultando em:

$$
c(t)=\left\{\begin{array}{l}
41,03+0,33 t, \text { se } L F<200 \\
29,83+0,39 t, \text { se } L F \geq 200
\end{array}\right.
$$

Custo mensal com o sistema Claro 3G e Skype:

$$
\begin{gathered}
c(t)=(1300 \times 0,09)+99,90+\frac{80,00}{12}+(0,7 \times 0,08) t+(0,3 \times 0,52) t \\
c(t)=223,57+0,21 t
\end{gathered}
$$




\subsection{Métodos Quantitativos de Análise Financeira}

\subsubsection{Análise do ponto de equilíbrio}

A partir dos modelos de custo da seção anterior, foram calculadas as diferenças entre os custos de utilização de ambos os sistemas em função da escala de utilização. Os resultados podem ser conferidos na Tabela 2.

Tabela 2 - Diferença de custos em função da escala de utilização

\begin{tabular}{cccc}
\hline $\begin{array}{c}\text { Escala de } \\
\text { utilização }\end{array}$ & $\begin{array}{c}\text { Custo com Claro 3G e } \\
\text { Skype }\end{array}$ & $\begin{array}{c}\text { Custo com linha } \\
\text { telefônica }\end{array}$ & $\begin{array}{c}\text { Diferença entre } \\
\text { sistemas }\end{array}$ \\
\hline 250 & $\mathrm{R} \$ 276,07$ & $\mathrm{R} \$ 127,33$ & $\mathrm{R} \$(148,74)$ \\
500 & $\mathrm{R} \$ 328,57$ & $\mathrm{R} \$ 224,83$ & $\mathrm{R} \$(103,74)$ \\
750 & $\mathrm{R} \$ 381,07$ & $\mathrm{R} \$ 322,33$ & $\mathrm{R} \$(58,74)$ \\
1000 & $\mathrm{R} \$ 433,57$ & $\mathrm{R} \$ 419,83$ & $\mathrm{R} \$(13,74)$ \\
1250 & $\mathrm{R} \$ 486,07$ & $\mathrm{R} \$ 517,33$ & $\mathrm{R} \$ 31,26$ \\
1500 & $\mathrm{R} \$ 538,57$ & $\mathrm{R} \$ 614,83$ & $\mathrm{R} \$ 76,26$ \\
1750 & $\mathrm{R} \$ 591,07$ & $\mathrm{R} \$ 712,33$ & $\mathrm{R} \$ 121,26$ \\
2000 & $\mathrm{R} \$ 643,57$ & $\mathrm{R} \$ 809,83$ & $\mathrm{R} \$ 166,26$ \\
2250 & $\mathrm{R} \$ 696,07$ & $\mathrm{R} \$ 907,33$ & $\mathrm{R} \$ 211,26$ \\
\hline
\end{tabular}

Fonte: Dados calculados a partir dos modelos da seção 5.1 .

Conforme apresentado na Tabela 1, a redução de custo com a adoção do sistema Claro 3G com Skype é proporcional à escala de utilização do sistema. Quanto maior for a escala de utilização do sistema, mais viável o sistema Claro 3G com Skype se torna em comparação com o sistema com linha telefônica.

O ponto de equilíbrio foi calculado igualando-se os modelos para o custo desenvolvidos na seção 5.1:

$$
\begin{aligned}
& 223,57+0,21 t=29,83+0,39 t \\
& t=1076,33 \text { minutos }
\end{aligned}
$$

Analisando o resultado, verifica-se que a adoção do sistema Claro 3G com Skype traz economia de custo para a empresa quando a escala de utilização for superior a 1076,33 minutos por mês. 
De acordo com o tempo total em ligações consumido pela empresa (3600 min), a economia esperada com a implantação do sistema Claro 3G com Skype corresponderia a aproximadamente $\mathrm{R} \$ 454,26$ mensais, além dos benefícios adquiridos com a internet de banda larga.

\subsubsection{Payback}

No caso do sistema Claro 3G com Skype, o investimento necessário na compra de equipamentos é a compra de um modem USB, que a Claro disponibiliza em sua loja on-line com preços a partir de $R \$ 116,00$; e a compra de um fone de ouvido com microfone, com valor estimado de $R \$ 25,00$; o que totaliza um investimento total de $\mathrm{R} \$ 141,00$. Considerando a economia obtida com a implantação deste sistema, de $\mathrm{R} \$ 454,26$ mensais, prevista pela análise do ponto de equilíbrio, o cálculo do payback fica:

$$
\text { Payback }=\frac{\mathrm{R} \$ 141,00}{\mathrm{R} \$ 454,26 / \mathrm{mês}} \approx 0,31 \text { mês }
$$

Verifica-se que a empresa começará com um déficit de $R \$ 141,00$, que será liquidado no mesmo mês de implantação do novo sistema.

\subsubsection{Valor Presente Líquido (VPL)}

$\mathrm{Na}$ análise do valor presente líquido, foi considerada a taxa de desconto de $11,75 \%$ a.a. (0,98\% a.m.), conforme a Selic, que é a taxa básica de juros da economia. O cálculo do VAL foi calculado com base em um horizonte de 6 meses estipulado arbitrariamente, conforme abaixo:

$$
\begin{gathered}
\mathrm{VPL}=\frac{(141,00)}{(1,0098)^{0}}+\frac{454,26}{(1,0098)^{1}}+\frac{454,26}{(1,0098)^{2}}+\frac{454,26}{(1,0098)^{3}}+\frac{454,26}{(1,0098)^{4}}+\frac{454,26}{(1,0098)^{5}}+\frac{454,26}{(1,0098)^{6}} \\
\mathrm{VPL}=2493,46
\end{gathered}
$$

O valor atual líquido positivo de $R \$ 2493,46$ significa que os ganhos do projeto cobrem o custo de capital da empresa, considerando a taxa de 0,98\% a.m.. 


\subsubsection{Taxa interna de retorno (TIR)}

A TIR foi calculada a partir da equação abaixo:

$$
\frac{(141,00)}{(1+i)^{0}}+\frac{454,26}{(1+i)^{1}}+\frac{454,26}{(1+i)^{2}}+\frac{454,26}{(1+i)^{3}}+\frac{454,26}{(1+i)^{4}}+\frac{454,26}{(1+i)^{5}}+\frac{454,26}{(1+i)^{6}}=0
$$

Com a ajuda de um sistema algébrico computacional, foi encontrado $\mathrm{i}=$ 3,2211 , que é a taxa interna de retorno (322,11\% a.m.). Interpretando esta taxa, constata-se que o capital investido $(R \$ 141,00)$ rende a uma taxa de juros compostos a $322,11 \%$ a.m. durante o período considerado (6 meses).

Uma das formas de analisar um investimento é confrontar a TIR com a taxa média de atratividade (TMA). A TMA é uma taxa associada ao baixo risco, na qual um investidor pode investir sobras de caixa e conseguir retornos financeiros.

Nesta análise, a TMA adotada foi à taxa Selic. Comparando a taxa interna de retorno (322,11\% a.m.) com a taxa Selic $(11,75 \%$ a.a.), verificou-se que a TIR é muito maior que a TMA, concluindo que o projeto é viável segundo este critério de decisão.

\section{CONSIDERAÇÕES FINAIS}

Neste trabalho buscou-se analisar a importância da análise de viabilidade econômica de projetos para a implantação de TIC em uma empresa do setor industrial alimentício, realizando-se para este fim um estudo de caso no qual foram comparados um sistema de VoIP (Claro 3G com Skype) e um sistema de linha telefônica (serviço Embratel com plano Sim 21 plus).

A análise de viabilidade econômica mostrou-se viável para o caso estudado, onde a escala de utilização do serviço pela empresa justifica a substituição do sistema de telefonia anterior (com menor custo fixo e maiores custos variáveis) pelo sistema de comunicação com VolP (com maior custo fixo e menores custos variáveis). Considerando-se os métodos de análise de viabilidade econômica apresentados na seção 5, pode-se notar que o critério é considerado viável segundo o critério de viabilidade de todos os métodos, o que indica grande vantagem na adoção deste tipo de sistema quando se apresenta grande escala de utilização do mesmo. 
Ressalta-se que foi indicada a implantação do sistema de VolP na empresa estudada, tendo em vista os resultados promissores obtidos pelos métodos de análise financeira aqui utilizados, e considerando também os benefícios a serem adquiridos com o uso de Internet de banda larga.

É importante destacar que a empresa em estudo (assim como a maior parte das empresas pertencentes ao setor industrial alimentício), trabalha com insumos perecíveis, que apresentam alta sazonalidade e que têm processos de conservação onerosos. As características destes insumos possibilitam sua estocagem somente durante curtos períodos de tempo, fazendo com que a integração da cadeia de suprimentos seja preponderante para a sobrevivência da empresa. Assim, esta integração constitui-se num dos grandes desafios enfrentados pela empresa, que objetiva que estes insumos estejam disponíveis e em condições adequadas para o seu processo de produção.

Neste contexto, destaca-se que as TIC podem proporcionar grande auxílio ao dinamizar os fluxos de informação na cadeia produtiva, aumentar a velocidade e reduzir os custos de comunicação, sendo que bons projetos para a implantação destas tecnologias tornam-se fundamentais para que uma empresa se mantenha competitiva frente a seus concorrentes.

Para trabalhos futuros, menciona-se aqui a possibilidade de obtenção de resultados interessantes através da realização de estudos similares analisando-se uma maior variedade de sistemas de comunicação por voz, o que inclui a utilização de uma maior gama de programas de comunicação via VolP, outros serviços para acesso a Internet e diferentes planos de telefonia fixa. Outras possibilidades seriam a utilização de métodos multicriteriais, para o estudo conjunto dos critérios (e não somente o critério custo) que envolvem a implantação de um novo sistema de comunicação; e/ou a utilização da Teoria das Opções Reais, para a avaliação dos riscos que envolvem cada decisão (ao longo do tempo) durante o processo de análise e implantação do projeto.

Finalmente, ressalta-se que um projeto detalhado, com o auxílio de métodos que avaliem os riscos e benefícios de um investimento, é de fundamental importância para o sucesso dos projetos para implantação de TIC, colaborando fortemente para a realização de projetos tecnológicos bem sucedidos. 


\section{REFERÊNCIAS}

ABIA - ASSOCIAÇÃO BRASILEIRA DA INDÚSTRIA DE ALIMENTOS. Indústria da alimentação: principais indicadores econômicos. São Paulo: ABIA, 2008.

BERNERS-LEE, T.; CAILLIAU, A. L.; NIELSEN, H. F.; SECRET, A. The World-Wide Web. Communications of the ACM, v. 37, n. 8, p. 76-82, ago. 1994.

BRITTO, W. S. F. Analise da viabilidade financeira da agricultura orgânica versus a agricultura convencional: o caso da manga no submédio do Vale do São Francisco. In: CONGRESSO DA SOCIEDADE BRASILEIRA DE ECONOMIA E SOCIOLOGIA RURAL, 42., 2004, Cuiabá. Anais... SOBER, 2004.

BRUNI, A. L.; FAMÁ, R.; SIQUEIRA, J. O. Análise do risco na avaliação de projetos: Uma aplicação do método de Monte Carlo. Caderno de Pesquisas em Administração, São Paulo, v. 1, n. 6, p. 1-14, 1998.

CASTELLANI, M. R. Cultura organizacional e tecnologia da informação: um estudo do uso da Internet na atividade de pesquisa em duas unidades da USP. São Paulo, SP: USP, 1998.

COLANGELO FILHO, L. C. Implantação de sistemas ERP: um enfoque de longo prazo. São Paulo: Atlas, 2001.

CUNHA, R. J. S. W. Ferramenta para comunicação VOIP usando o padrão H.323 em redes com servidores NAT. Florianópolis, SC: UFSC, 2004.

DIAS, L. Número de usuários da Internet nas residências permaneceu estável. 30 mai. 2009. Disponível em: <http://www.vooz.com.br/noticias/numero-de-usuariosda-internet-nas-residencias-permaneceu-estavel-5154.html>. Acesso em: 6 jul. 2009.

DIAS, R. M.; PITASSI, C.; JOIA, L. A. Gestão integrada da cadeia de suprimento. Journal of Business Logistics, v. 18, n. 2, p. 31, 1997.

EMBRATEL. Profissionais liberais e microempresas: adesão a planos de tarifas. Disponível em: < http://www.embratel.com.br/Embratel02/cda/portal/0,2997,REP3 192,00.html >. Acesso em: 29 fev. 2009.

EMPRESA CLARO DE TELEFONIA. Banda larga 3G. Disponível em: < http://www.claro.com.br/portal/precos e tarifas.php>. Acesso em: 29 fev. 2009.

FONTES, C. A. Usos e efeitos da Internet na prática bibliográfica: estudo exploratório junto ao Sistema Integrado de Bibliotecas da Universidade de São Paulo. São Paulo, SP: USP, 2001.

GAITHER, N.; FRAZIER, G. Administração da produção e operações. 8. ed. São Paulo: Pioneira Thomson, 2002. 
GODOY, A. S. Introdução à pesquisa qualitativa e suas possibilidades. Revista de Administração de Empresas, [S.I], v. 35, n. 3, maio/jun. 1995.

GONÇALVES, T. J. M. Planejamento, implementação e aplicação de um sistema web de coleta de dados para o auxílio à decisão. Campos dos Goytacazes, RJ: UENF, 2010.

JUNG, C. F. Metodologia para pesquisa e desenvolvimento: aplicada a novas tecnologias, produtos e processos. Rio de Janeiro: Axcel Books, 2004.

LA ROVERE, R. L. As pequenas e médias empresas na economia do conhecimento: implicações para políticas de inovação. In: LASTRES, H. M. M.; ALBAGLI, S. (Orgs.) Informação e globalização na era do conhecimento. Rio de Janeiro: Campus, 1999 p.145-163.

LAMBERT, D. M.; COOPER, M. C. Issues in supply chain management. The International Journal of Logistics Management. Flórida, v. 29, p. 65-83, 2000.

LAUDON, K. C; LAUDON, J. P. Sistemas de informações gerenciais. São Paulo: Prentice Hall, 2004. 562 p.

LI, S. Developing measures of supply chain management performance. In: ANNUAL MEETING DECISION SCIENCES INSTITUTE, San Diego, California, nov. 23-26, 2002, Proceedings... California, 2002.

MONTEIRO, R. F. Implementação de transporte robusto de voz em redes baseadas em protocolos IP. Belo Horizonte, MG: UFMG, 2000.

NARASHIMHAN, R.; JAYARAM, J. Causal linkage in supply chain management: an exploratory study of North American manufacturing firms, Decision Sciences, v. 29, n. 3, p. 579-605, 1998.

OGGH, F. A mãe de todas as páginas em html. 17 dez. 2001. Disponível em: $<$ http://webinsider.uol.com.br/index.php/2001/12/17/a-mae-de-todas-as-paginas-emhtml>. Acesso em: 6 jul. 2009.

PERES, A. A. C. et al. Análise econômica de sistemas de produção a pasto para bovinos no município de Campos dos Goytacazes - RJ. Revista Brasileira de Zootecnia, v.33, n.6, p.1557-1563, 2004.

RUTHFIELD, S. The Internet history and development: from wartime tool to the fish-cam. ACM Crossroads. New York, jan. 2001. Disponível em: <http://www.acm.org/crossroads/xrds2-1/inet-history.html>. Acesso em: 22 abr. 2009.

SANTOS, V. M. Vantagens e dificuldades de integração com fornecedores: um estudo de caso em uma empresa do ramo automobilístico. Pedro Leopoldo, MG: FIPL,2010. 
SKYPE. Tarifas SkypeOut. Disponível em: < http://www.skype.com/intl/pt/ products/skypeout/rates/>. Acesso em: 29 fev. 2009.

STRASSMANN, Paul. A. Will big spending on computers guarantee profitability? Datamation, fev. 1997.

TURBAN, E.; RAINER, R. K.; POTTER, E. E. Administração de tecnologia da informação: teoria e prática. 3. ed. São Paulo: Campus, 2005.

WOILER, S.; MATHIAS, W. F. Projetos: planejamento, elaboração e análise. São Paulo: Atlas, 1996. 294 p.

WOOD, A. Extending the supply chain: strengthening links with it. Chemical Week, v. 159, n. 25, p. 25-26, 1997.

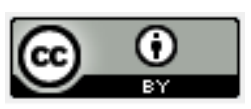

Artigo recebido em 05/02/2010 e aceito para publicação em 17/08/2011. 